\title{
Morphology Changes of Co Catalyst Nanoparticles at the Onset of Fischer-Tropsch Synthesis
}

\author{
Kristin Høydalsvik, ${ }^{\dagger}$ Jostein B. Fløystad, ${ }^{\dagger}$ Alexey Voronov, ${ }^{\ddagger}$ Georg J. B. Voss, ${ }^{\ddagger}$ Morteza Esmaeili, ${ }^{\dagger}$ \\ Jan Kehres, ${ }^{\S}$ Håvard Granlund, ${ }^{\dagger}$ Ulla Vainio, $"$ Jens W. Andreasen, ${ }^{\perp}$ Magnus Rønning, \\ and Dag W. Breiby*, $\dagger$ \\ ${ }^{\dagger}$ Department of Physics and ${ }^{\ddagger}$ Department of Chemical Engineering, Norwegian University of Science and Technology (NTNU), \\ NO-7491 Trondheim, Norway \\ ${ }^{\S}$ Center of Individual Nanoparticle Functionality, Department of Physics, Technical University of Denmark, DK-2800 Kongens \\ Lyngby, Denmark \\ "Hasylab at Deutsches Elektronen-Synchrotron (DESY), 22607 Hamburg, Germany \\ ${ }^{\perp}$ Department of Energy Conversion and Storage, Technical University of Denmark, DK-4000 Roskilde, Denmark
}

ABSTRACT: Cobalt nanoparticles play an important role as catalysts for the FischerTropsch synthesis, which is an attractive route for production of synthetic fuels. It is of particular interest to understand the varying conversion rate during the first hours after introducing synthesis gas $\left(\mathrm{H}_{2}\right.$ and $\left.\mathrm{CO}\right)$ to the system. To this end, several in situ characterization studies have previously been done on both idealized model systems and commercially relevant catalyst nanoparticles, using bulk techniques, such as X-ray powder diffraction and X-ray absorption spectroscopy. Since catalysis takes place at the surface of the cobalt particles, it is important to develop methods to gain surface-specific structural information under realistic processing conditions. We addressed this challenge using small-angle X-ray scattering (SAXS), a technique exploiting the penetrating nature of Xrays to provide information about particle morphology during in situ experiments. Simultaneous wide-angle X-ray scattering was used for monitoring the reduction from oxide to catalytically active metal cobalt, and anomalous SAXS was used for distinguishing the cobalt particles from the other phases present. After introducing the synthesis gas, we found that the slope of the scattered intensity in the Porod region increased significantly, while the scattering invariant remained essentially constant, indicating a change in the shape or surface structure of the particles. Shape- and surface change models are discussed in light of the experimental results, leading to an improved understanding of catalytic nanoparticles.

\section{INTRODUCTION}

The Fischer-Tropsch synthesis (FTS) is a set of chemical reactions that forms hydrocarbon chains from a mixture of $\mathrm{CO}$ and $\mathrm{H}_{2}$. The product can be upgraded to petroleum substitutes, for example synthetic diesel. ${ }^{1}$ Typical commercial FTS catalysts consist of cobalt nanoparticles of diameter $\sim 20 \mathrm{~nm}$ dispersed on a porous support material, ${ }^{2}$ such as $\gamma$-alumina. Optimal particle size, temperature, and pressure are required for obtaining high activity and high selectivity to long-chain hydrocarbons. The reaction output is dependent on the temperature and the pressure in the reactor cell; the standard industrial process operates at $T \approx 220^{\circ} \mathrm{C}$ and pressure of $25-$ $45 \mathrm{bar}$, conditions favorable for producing waxes. ${ }^{1}$ At ambient pressure the $\mathrm{CO}$ conversion is still high, but the products are predominantly short molecules, thus tending to remain in the gas phase.

The FTS shows an initial stage lasting a few hours where the conversion rate increases to a high level, followed by a much slower decrease of the reaction rate that continues on a time scale of days and months. ${ }^{3}$ Understanding the mechanisms behind this behavior is of high commercial and academic interest. Tsakoumis et al. ${ }^{3}$ combined in situ X-ray absorption spectroscopy (XAS) and X-ray powder diffraction to investigate the cobalt catalyst nanoparticles during the FTS synthesis. The deactivation was detected by mass spectrometry, but no apparent changes in the X-ray signal could be observed, and thus no observable bulk-related changes, emphasizing the need for in situ surface characterization of the cobalt particles during FTS. Schulz et al. ${ }^{4}$ measured changes in activity and selectivity during the initial hours of FTS and noticed that since CO chemisorbs strongly on cobalt, the reaction of $\mathrm{CO}$ with the metal surface can be assumed to induce surface restructuring. Wilson and de Groot reported restructuring of a $\mathrm{Co}(0001)$ surface to triangular cobalt islands when exposed to $\mathrm{CO}$ hydrogenation conditions. ${ }^{5}$ The CO-induced surface restructuring was also confirmed by polarization modulation-reflection absorption infrared spectroscopy. ${ }^{6}$

Received: May 27, 2013

Revised: December 20, 2013 
The Fischer-Tropsch synthesis has previously been studied using a variety of techniques, including Auger electron spectroscopy and electron energy loss spectroscopy, ${ }^{7,8}$ temperature programmed studies, ${ }^{9-11}$ infrared spectroscopy, ${ }^{11,12} \mathrm{X}$-ray powder diffraction, ${ }^{3,13}$ transmission electron microscopy (TEM) ${ }^{14,15}$ X-ray absorption spectroscopy, ${ }^{3,13,16}$ thermogravimetric analysis, ${ }^{9,10,17} \mathrm{X}$-ray photoelectron spectroscopy, ${ }^{10}$ mass spectrometry, ${ }^{3,12,13}$ scanning tunneling microscopy, ${ }^{18,19}$ and Mössbauer emission spectroscopy. ${ }^{17}$ Previously, we have used environmental TEM for studying the reduction of cobalt particles prepared in the same way as the samples in this study, ${ }^{15}$ with the only difference being that the cobalt particles were supported on $\alpha$-alumina instead of $\gamma$-alumina. However, none of the above techniques are capable of yielding surfacespecific structural information under relevant processing conditions. Consequently, it is obvious that a technique sensitive to changes of the surface of the Co catalyst particles is in demand. Because the FTS conversion from gas to liquid necessarily needs a certain pressure, the FTS processes cannot be studied using techniques requiring high vacuum, such as TEM.

Due to their penetrating nature, hard X-rays of energy $\gtrsim 5 \mathrm{keV}$ can be used for studying processes that require high pressure and bulky sample environments. Small-angle X-ray scattering (SAXS) denotes the elastic scattering of X-rays by inhomogeneities in the electron density in the size range from $\sim 1$ to $100 \mathrm{~nm}$, typically representing particles or pores. From SAXS patterns, structural information about these electron density inhomogeneities can be obtained, ${ }^{20}$ and SAXS has previously been used for monitoring changing size, ${ }^{21}$ shape, ${ }^{22}$ and roughness ${ }^{23}$ of particles in situ. Preux et al. have used SAXS for monitoring expansion and faceting of viral capsids, ${ }^{24}$ and Braun et al. have used ultra-small-angle X-ray scattering (USAXS) for studying cobalt precipitates in silica aerogels before and after reduction. ${ }^{25}$ Grazing-incidence small-angle Xray scattering (GISAXS) has been used, for example, for monitoring the evolution in the size distribution of nanoparticles deposited on surfaces in situ, ${ }^{26-29}$ and for quantitative analysis of faceted islands on surfaces. ${ }^{30}$

Here, we apply in situ SAXS to Co nanoparticles supported on $\gamma$-alumina under realistic FTS conditions and find that the Porod exponent changes markedly upon switching from hydrogen $\left(\mathrm{H}_{2}\right)$ to synthesis gas $\left(\mathrm{H}_{2}+\mathrm{CO}\right)$. This is an intriguing result in its own right, and a discussion of this result in the light of possible underlying mechanisms is given.

\section{SAXS ANALYSIS FOR HETEROGENEOUS CATALYSIS}

A schematic SAXS setup is shown in Figure 1. For a monodisperse collection of identical randomly oriented spheres, the SAXS intensity can be written as ${ }^{31}$

$$
I(Q) \propto \frac{\mathrm{d} \Sigma}{\mathrm{d} \Omega}(Q)=n \Delta \rho^{2} V_{\mathrm{p}}^{2}|F(Q)|^{2} S(Q)
$$

where $\mathrm{d} \Sigma(Q) / \mathrm{d} \Omega$ denotes the differential scattering crosssection for a spherically symmetric collection of particles normalized by irradiated volume. The wave-vector transfer is $Q$ $=|\mathrm{Q}|=(4 \pi \sin \theta) / \lambda$, where $\theta$ is half the scattering angle, and $\lambda$ the wavelength of the incoming X-rays. $n$ is the number density of scattering objects in the sample, and $V_{\mathrm{p}}$ is the volume of one scattering object. $\Delta \rho$ is the difference in scattering length density, or "contrast", which is proportional to the difference in electron densities of the different phases of the sample, in the a) View along beam direction

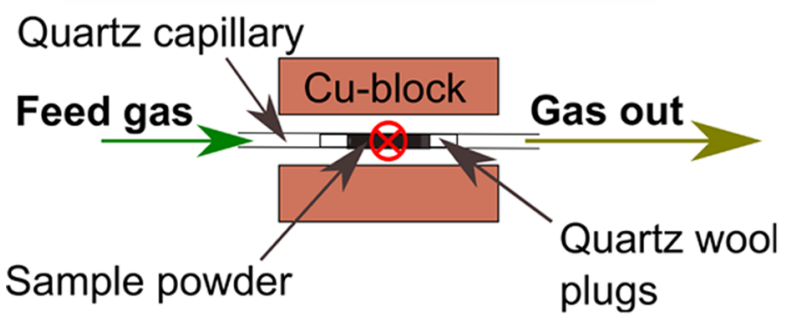

b) Side view

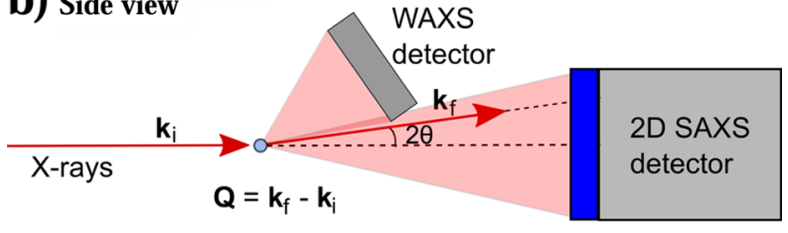

c) Measured SAXS pattern

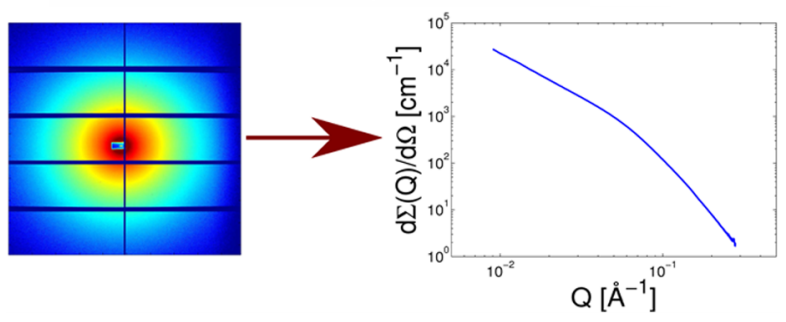

Figure 1. Schematic figure of the experimental setup. (a) The quartz capillary containing the sample powder was mounted between $\mathrm{Cu}$ blocks for heating and connected to gas lines. The red cross indicates the X-ray beam going into the paper plane. (b) Side-view of the SAXS and WAXS geometry. (c) An example of measured data; the raw data are on the left, and the 1D scattering pattern obtained after azimuthal averaging is on the right.

present case including cobalt, cobalt oxides, the $\gamma$-alumina matrix, and pores. $\Delta \rho$ depends also on the X-ray wavelength through energy-dependent dispersion corrections. ${ }^{32} F(Q)$ is the form factor amplitude which contains information about the particle shape; for spheres it can be calculated analytically to be $F_{\text {sphere }}(Q)=3[\sin (Q R)-Q R \cos (Q R)] /(Q R)^{3}$, where $R$ is the particle radius. ${ }^{33}$ The structure factor $S(Q)$ describes the interference of scattering from different particles and thus contains information about the spatial correlations between the particles. For dilute systems, $S(Q)=1$ and can be neglected. ${ }^{31}$

SAXS patterns tend to exhibit a linear "Porod" region at comparably large $Q$ when presented on a double-logarithmic plot. The scattering intensity, $I(Q) \propto|F(Q)|^{2}$, calculated in the limit $Q R \gg 1$, yields ${ }^{34,35}$

$$
I(Q) \propto Q^{-\alpha}
$$

The power-law exponent $\alpha$, known as the Porod exponent, is related to the surface structure and to the shape of the particles: $\alpha=1$ for thin rods, $\alpha=2$ for thin discs, and $\alpha=4$ for smooth 3D objects. Moreover, $1<\alpha<3$ for mass fractals, $3<\alpha<4$ for surface fractals, and $\alpha>4$ for particles with a continuous interface transition. ${ }^{36,37}$ Schmidt et al. have studied porous silicas ${ }^{37}$ and observed $\alpha>4$, which they ascribe to the interface between the pore and the matrix being continuous rather than abrupt. Implementing a graded interface can be done analytically by convoluting the density function $\rho(r)$ with a 
Table 1. Summary of Experimental Parameters Used in the Current and Related Studies ${ }^{a}$

\begin{tabular}{|c|c|c|c|c|c|c|}
\hline & & \multicolumn{3}{|c|}{ current SAXS study } & \multirow[b]{2}{*}{ Rønning et al. ${ }^{13}$} & \multirow[b]{3}{*}{ Tsakoumis et $\mathrm{al}^{3}$} \\
\hline & & \multicolumn{2}{|c|}{$16 \mathrm{keV}$ expt } & \multirow{2}{*}{$\frac{7 \mathrm{keV} \text { expt }}{\text { sample A }}$} & & \\
\hline & & sample A & sample B & & FT1/FT2 & \\
\hline \multirow[t]{2}{*}{ sample } & $\begin{array}{l}\text { catalyst } \\
\text { composition }\end{array}$ & $\begin{array}{c}20 \text { wt } \% \mathrm{Co} \\
1 \text { wt } \% \operatorname{Re}\end{array}$ & $\begin{array}{l}20 \text { wt } \% \mathrm{Co}, \\
0.5 \text { wt } \% \operatorname{Re}\end{array}$ & $\begin{array}{c}20 \text { wt } \% \mathrm{Co} \\
1 \text { wt } \% \operatorname{Re}\end{array}$ & $\begin{array}{c}20 \text { wt } \% \mathrm{Co} \\
1 \text { wt } \% \operatorname{Re}\end{array}$ & $\begin{array}{c}20 \text { wt \% Co, } \\
1 \text { wt } \% \operatorname{Re}\end{array}$ \\
\hline & support & $\gamma-\mathrm{Al}_{2} \mathrm{O}_{3}$ & $\gamma-\mathrm{Al}_{2} \mathrm{O}_{3}$ & $\gamma-\mathrm{Al}_{2} \mathrm{O}_{3}$ & $\gamma-\mathrm{Al}_{2} \mathrm{O}_{3}$ & $\gamma-\mathrm{Al}_{2} \mathrm{O}_{3}$ \\
\hline \multirow[t]{5}{*}{ reduction } & $\begin{array}{l}\text { ramp rate } \\
(\mathrm{deg} / \mathrm{min})\end{array}$ & 3 & 3 & 3 & 3 & 3 \\
\hline & set point $T\left({ }^{\circ} \mathrm{C}\right)$ & 350 & 350 & 350 & 400 & 400 \\
\hline & gas used & $\mathrm{H}_{2}$ & $\mathrm{H}_{2}$ & $\mathrm{H}_{2}$ & $\mathrm{H}_{2}$ & $\mathrm{H}_{2}$ \\
\hline & gas flow $(\mathrm{mL} / \mathrm{min})$ & 2.5 & 2.5 & 2.5 & 2.5 & 2.5 \\
\hline & time at set point & 4 & 4 & 4 & 4 & 4 \\
\hline \multirow{4}{*}{$\begin{array}{l}\text { pressure } \\
\text { increase }\end{array}$} & $T\left({ }^{\circ} \mathrm{C}\right)$ & - & - & 180 & 170 & 180 \\
\hline & pressure (bar) & - & - & $1-10$ & $1-10$ & $1-18$ \\
\hline & gas used & - & - & $\mathrm{H}_{2}$ & $\mathrm{H}_{2} / \mathrm{CO}$ & $\mathrm{H}_{2} / \mathrm{CO}$ \\
\hline & $T\left({ }^{\circ} \mathrm{C}\right)$ & $180-210$ & $180-210$ & $180-210$ & $170-210$ & $170-220$ \\
\hline \multirow[t]{3}{*}{ induction } & $\begin{array}{l}\text { ramp rate } \\
(\mathrm{deg} / \mathrm{min})\end{array}$ & 1 & 1 & 1 & - & 1 \\
\hline & gas used & $\mathrm{H}_{2} / \mathrm{CO}$ & $\mathrm{H}_{2} / \mathrm{CO}$ & $\mathrm{H}_{2} / \mathrm{CO}$ & $\mathrm{H}_{2} / \mathrm{CO}$ & $\mathrm{H}_{2} / \mathrm{CO}$ \\
\hline & TOS (h:min) & $1: 20$ & $2: 40$ & $3: 50$ & - & $7: 00$ \\
\hline \multirow[t]{2}{*}{ FTS cond. } & $T\left({ }^{\circ} \mathrm{C}\right)$ & 210 & 210 & - & 210 & 220 \\
\hline & TOS $(\mathrm{h})$ & 7 & 2 & - & 2 & 32 \\
\hline
\end{tabular}

${ }^{a}$ During the reduction, the set-point temperature was lowered in the current study to avoid sintering, as a temperature of $350{ }^{\circ} \mathrm{C}$ was found to be sufficient for reducing the sample.

Gaussian, ${ }^{38}$ which by the convolution theorem modifies the form factor amplitude $F(Q)$ in eq 1 to become

$$
F_{\text {graded }}(Q)=F(Q) \exp \left[-\left(\sigma_{\text {surf }} Q\right)^{2} / 2\right]
$$

Thus, on a double-logarithmic plot as in Figure 4, the enveloping slope becomes steeper as the standard deviation of the Gaussian distribution, $\sigma_{\text {surf }}$ increases. Changes in $\alpha$ can thus be attributed to changes in the surface morphology of the scattering objects. $^{33}$

Another main feature in classical SAXS analysis is the presence of a flat region for low $Q$, the so-called Guinier region, which yields the radius of gyration, $R_{\mathrm{g}} \cdot{ }^{33,34}$ However, for heterogeneous and polydisperse systems, as discussed here, this analysis is hardly reliable and will not be further elaborated upon here.

The scattering invariant,

$$
G=\int_{0}^{\infty} Q^{2} I(Q) d Q
$$

depends on the mean square fluctuation of electron density in the sample and not on the specific structure causing it. ${ }^{35}$ Consequently, for scattering objects only undergoing reshaping, such as faceting, $G$ should remain constant, while changes in densities or amount of material will cause $G$ to change. The integration limits for $Q$ are not feasible experimentally, and the commonly used solution is to extrapolate the measured SAXS patterns, by the Guinier analysis for low $Q$ and the power-law analysis for high $Q$. However, it was not possible to use the Guinier analysis here due to the presence of large cobalt agglomerates for which the low $Q$ flat region could not be determined from our SAXS measurements. Instead we use the approximation

$$
G^{*}=\int_{Q_{\min }}^{Q_{\max }} Q^{2} I(Q) \mathrm{d} Q
$$

i.e., integrating within the measured range. However, this approximation needs to be used with care, as changes in the scattering pattern could cause changes in $G^{*}$, even if $G$ itself is in fact constant. The scattering invariant for a multiphase system can be modeled by ${ }^{39}$

$$
G=2 \pi^{2} \sum_{i<j} \phi_{i} \phi_{j}\left(\rho_{\mathrm{s}, i}-\rho_{\mathrm{sl}, j}\right)^{2}
$$

where $\phi_{i}$ and $\phi_{j}$ are the volume fractions, and $\rho_{\mathrm{sl}, i}$ and $\rho_{\mathrm{sl}, j}$ are the scattering length densities, for phase $i$ and $j$, respectively.

In anomalous SAXS (ASAXS) experiments, the pronounced element-specific dispersion occurring near absorption edges are exploited for increased contrast. The scattered intensity from a two-phase system can be understood as a sum of three terms, ${ }^{40,41}$

$$
I(Q, E)=I_{11}(Q, E)+I_{22}(Q)+I_{12}(Q, E)
$$

where (1) represents the phase containing the element that has the absorption edge close to energy E. Calculating the difference between one measurement taken near the edge, and another measurement well below, yields $\Delta I(Q E)=$ $\alpha I_{11}(Q E)+\beta I_{12}(Q E)$, where $\alpha$ and $\beta$ are proportionality constants. Notice that we are left with the terms that contain phase $1\left(I_{11}\right.$ and $\left.I_{12}\right)$, while the term containing only phase 2 $\left(I_{22}\right)$ has been canceled out.

\section{EXPERIMENTAL SECTION}

The catalyst samples used in this study consist of rheniumpromoted cobalt particles supported on high surface area porous $\gamma-\mathrm{Al}_{2} \mathrm{O}_{3}$ (Puralox SCCa from Sasol $\mathrm{GmbH}$ ). The nanoparticles were prepared by coimpregnation of $\mathrm{Co}\left(\mathrm{NO}_{3}\right)_{2}$. $6 \mathrm{H}_{2} \mathrm{O}$ and $\mathrm{HReO}_{4}$ to give 20 wt \% Co and either 1 wt \% or 


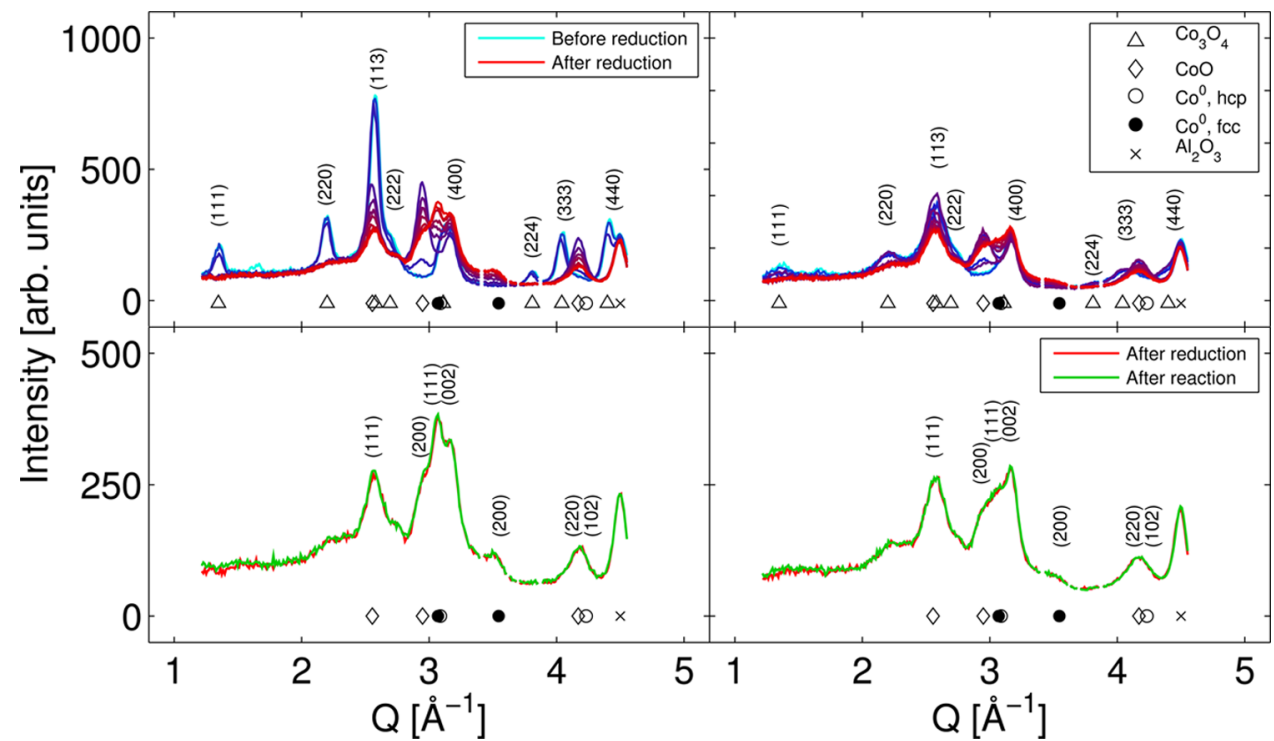

Figure 2. WAXS patterns measured during the experiment. The top panels show every tenth measurement during reduction, and the color indicates how far into the experiment the measurement was performed, from cyan for unreduced via blue for partly reduced to red for reduced sample. The $h k l$-indices for $\mathrm{Co}_{3} \mathrm{O}_{4}$ peaks are indicated. The bottom panels show the last reduction measurement (red) and the last measurement at FTS conditions (green). Here the $\mathrm{CoO}$ and the $\mathrm{Co}^{0}-\mathrm{fcc}$ and - hcp peaks are labeled.

(a)

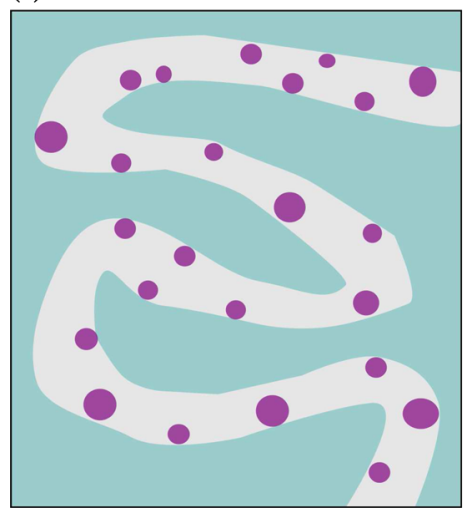

(b)

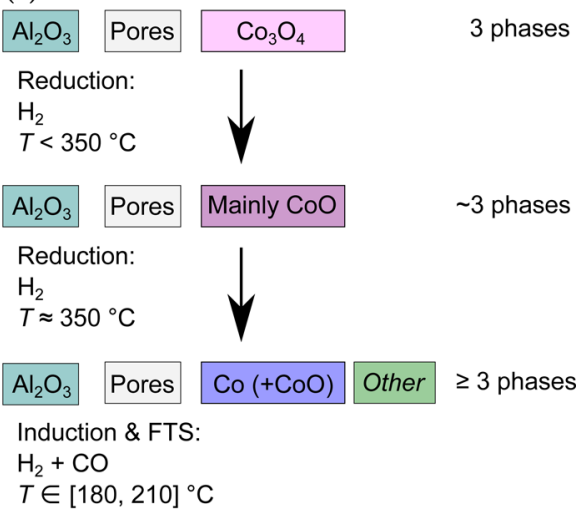

Figure 3. (a) Schematic drawing of the constituent phases, with porous alumina and polydisperse nanoparticles. (b) Schematic showing the different stages of the experiment. When the temperature reaches $350{ }^{\circ} \mathrm{C}$ during the reduction, most of the Co-containing particles have been reduced. Even after several hours of reduction, a fraction of the cobalt remain in the $\mathrm{CoO}$ phase. ${ }^{13}$ The other possible phases are discussed in the main text.

0.5 wt \% Re for samples $\mathrm{A}$ and $\mathrm{B}$, respectively, with the remaining $\sim 80 \%$ being $\gamma$-alumina. The samples were dried in air at $120{ }^{\circ} \mathrm{C}$ for $3 \mathrm{~h}$ and calcined in air at $300{ }^{\circ} \mathrm{C}$ for $16 \mathrm{~h}$. The sample powders were sieved, and the fraction with grain-sizes between 53 and $90 \mu \mathrm{m}$ was used for the experiments. ${ }^{13}$ Using $\mathrm{H}_{2}$ chemisorption, we have performed particle size measurements, supported by TEM, that gave the diameter of the cobalt particles in sample A as $10 \pm 1$ and $5 \pm 1 \mathrm{~nm}$ for sample B. ${ }^{14}$ For the SAXS experiments, the sample powder was loaded into a quartz capillary tube (10 $\mu \mathrm{m}$ wall thickness; Markröhrchen, Hilgenberg $\mathrm{GmbH}$ ) which was mounted in the in situ sample cell, $^{42}$ cf. Figure 1.

As prepared, the cobalt nanoparticles are in the catalytically passive oxidized state $\left(\mathrm{Co}_{3} \mathrm{O}_{4}\right)$ and thus have to be reduced to metallic cobalt in order to provide active sites for catalysis. ${ }^{43}$ This was done in situ in $\mathrm{H}_{2}$ atmosphere while heating the sample using a heat ramp of $3{ }^{\circ} \mathrm{C} / \mathrm{min}$ to $350{ }^{\circ} \mathrm{C}$, and the temperature was kept stable at $350{ }^{\circ} \mathrm{C}$ for $4 \mathrm{~h}$. Then the temperature was lowered to $180{ }^{\circ} \mathrm{C}$ at a rate of $5{ }^{\circ} \mathrm{C} / \mathrm{min}$. For the experiment where the sample environment was pressurized, the pressure was increased gradually to $10 \mathrm{bar}$, still using $\mathrm{H}_{2}$ as feed gas. The feed-gas was subsequently changed to synthesis gas consisting of hydrogen and carbon monoxide in molecular ratio $\mathrm{H}_{2} / \mathrm{CO}=2.1$, mixed with $10 \mathrm{vol} \% \mathrm{He}$. The purity of the gases was 4.7 for $\mathrm{CO}, 5.0$ for $\mathrm{H}_{2}$, and 5.6 for He. After an induction period, the temperature was increased slowly $\left(1^{\circ} \mathrm{C} /\right.$ min) until reaching FTS conditions at $210{ }^{\circ} \mathrm{C}$ and kept stable for several hours. The experimental conditions are summarized in Table 1, where also parameters from related studies discussed in this article are listed. ${ }^{3,13}$

The X-ray scattering experiments were performed at beamline $\mathrm{B} 1$ of the DORIS III synchrotron storage ring of Hasylab at DESY. The main experiment was done using an Xray energy of $16.0 \mathrm{keV}$ and a sample-to-detector distance of $3575 \mathrm{~mm}$. The SAXS detector was a two-dimensional Pilatus $1 \mathrm{M}$ (Dectris) detector ${ }^{44}$ with $981 \times 1043$ pixels of size $172 \mu \mathrm{m} \times 172 \mu \mathrm{m}$. Simultaneous wide-angle X-ray scattering measurements were used to confirm the reduction of 

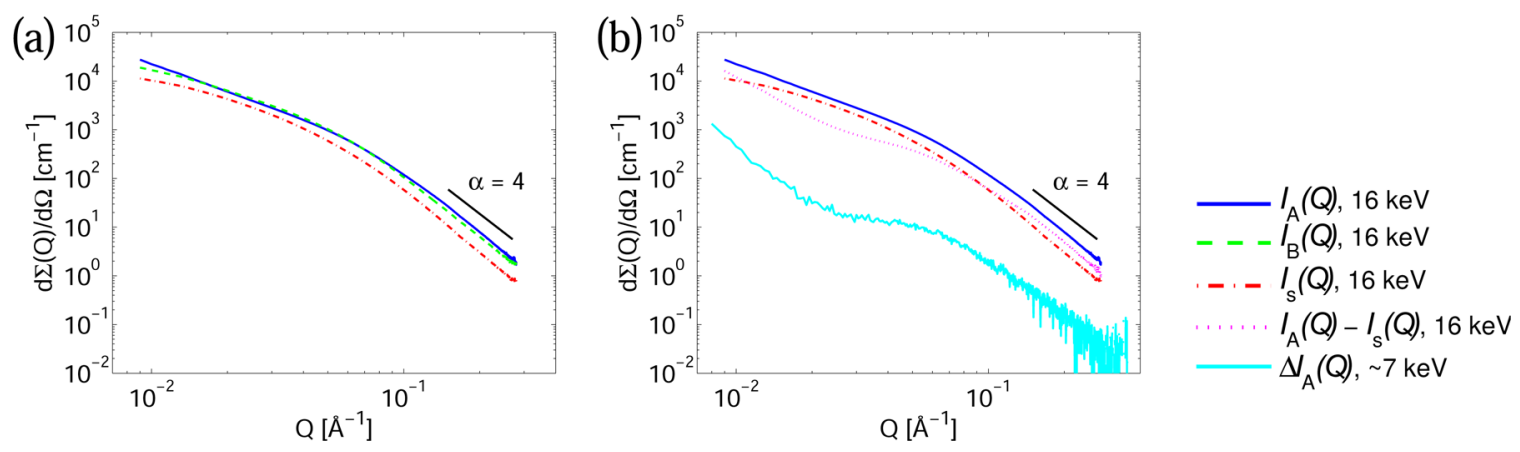

Figure 4. (a) The SAXS patterns from sample $A, I_{A}(Q)$, and sample $B, I_{B}(Q)$, measured after reducing the sample, and the $\gamma$-alumina support, $I_{s}(Q)$, measured under ambient conditions $(E=16 \mathrm{keV})$. The black straight line indicates the Porod region where the power-law exponent $\alpha$ was found by fitting a straight line to the SAXS pattern. (b) $I_{\mathrm{A}}(Q)$ and $I_{\mathrm{s}}(Q)$ measured at $E=16 \mathrm{keV}$, and the difference between the two. The cyan curve is the ASAXS difference curve for sample A, $\Delta I_{A}(Q)=I_{A}\left(Q E_{1}\right)-I_{A}\left(Q E_{2}\right)$, where $E_{1}=7509 \mathrm{eV}$ and $E_{2}=7694 \mathrm{eV}$, measured during reduction.

cobalt, using a one-dimensional Mythen II detector (Dectris). The outer diameter of the capillary tube used for containing the sample powder was $1.5 \mathrm{~mm}$. In a complementary experiment, several energies below the Co K-edge at $7709 \mathrm{eV}$ were used to obtain additional contrast from anomalous scattering for sample A. The distance between the sample and the SAXS detector was $1313 \mathrm{~mm}$, and the outer diameter of the capillary tube used was $0.7 \mathrm{~mm}$. In both cases the exposure time was 60 or $180 \mathrm{~s}$, depending on the required time resolution in the different periods. The obtained isotropic SAXS patterns were normalized by the incoming photon flux, as well as the transmission and thickness of the sample, integrated over the azimuth angle and calibrated to absolute intensity units $\left(\mathrm{cm}^{-1}\right)$ using a glassy carbon reference sample. ${ }^{45}$

\section{RESULTS}

Wide-angle scattering patterns measured during reduction with $\mathrm{H}_{2}$ gas are presented in Figure 2, showing that the $\mathrm{Co}_{3} \mathrm{O}_{4}$ peaks vanish and $\mathrm{Co}^{0}$ peaks appear. After reduction, the WAXS patterns remained unchanged. The constituent phases of the sample for each stage of the experiment are illustrated in Figure 3. The peak intensities measured for sample A are significantly higher than those for sample B before reduction, while they become comparable after reduction, suggesting that the crystallites of samples A and B become more similar.

Small-angle scattering patterns measured for the different samples after reduction are presented in Figure 4. It is readily realized that there are only a few features in the curves, and a detailed structural model thus cannot be deduced. In the figure we notice both for the two catalyst-containing samples and for the $\gamma$-alumina reference sample a power-law slope in the high- $Q$ region $\left(Q>0.1 \AA^{-1}\right)$ and a weak shoulder in the region $0.04 \AA^{-1}<Q<0.07 \AA^{-1}$. The position of the shoulder is related to the radii of gyration $R_{\mathrm{g}}$ of the scattering objects. In the present case this shoulder is hardly observable, which is due to overlapping of scattering signals from the polydisperse cobalt particles and the irregular pore structure of the alumina support. The size distributions for the different constituents are highly polydisperse, as well as overlapping, which precludes finding a unique solution for the actual size distributions. In this work, we therefore rely on the model-independent analysis described in the section on SAXS analysis. The difference in scattering intensity between the $\gamma$-alumina and the catalyst samples is slightly more pronounced at the lowest $Q$-values for sample A (Figure 4a), which might be ascribed to scattering from cobalt particle agglomerates, corroborating previous TEM measurements. $^{15}$

To better separate the contributions to the scattering signal, sample A has additionally been measured using ASAXS around the Co K-edge at $E_{\text {edge }}=7709 \mathrm{eV}$. For the ASAXS analysis we separate the sample material into two phases; ${ }^{46}$ cobalt (1) and porous alumina (2). The difference curve is obtained from the experimental data by subtracting the exposure taken close to the Co K-edge $\left(E_{2}=E_{\text {edge }}-15 \mathrm{eV}\right)$ from one made further below the edge $\left(E_{1}=E_{\text {edge }}-200 \mathrm{eV}\right), \Delta I_{\mathrm{A}}(Q)=I_{\mathrm{A}}\left(Q E_{1}\right)-$ $I_{\mathrm{A}}\left(Q, E_{2}\right)$. Notice that the difference curve, $\Delta I$, shown in Figure $4 \mathrm{~b}$, has a distinct shoulder at $Q \simeq 0.07 \AA^{-1}$. It is therefore reasonable to assume a significant contribution from the cobalt particles in this region, which is crucial in justifying the further analysis, as it suggests that changes observed in the Porod region can be ascribed to the, as compared to the $\gamma$-alumina pores, small Co particles.

It is seen in Figure 4 that the power-law exponent $\alpha$ has values near 4. Optimally, the SAXS patterns should have been measured over a larger $Q$-range in order to get accurate absolute values of $\alpha$, but for in situ experiments this is difficult to obtain. In this work, we mainly study changes in $\alpha$ as the in situ experiment proceeds, which can be done by using an experimentally more well-defined relative value of $\alpha$. Since it is reasonable to assume that the state of the cobalt particles is the same in all the experiments after reduction, as supported by the WAXS results (see Figure 2), we define a relative $\alpha$ as $\Delta \alpha=\alpha_{i}-\alpha_{0}$, where $\alpha_{i}$ is the value of $\alpha$ found for measurement $i$ and $\alpha_{0}$ is the measured $\alpha$ for the samples after reduction in $\mathrm{H}_{2}$ atmosphere, at $180{ }^{\circ} \mathrm{C}$.

The values obtained for $\Delta \alpha$ from the in situ experiments are plotted in Figure 5 as function of time and experimental conditions after the experiment started. For the catalysis to take place, the cobalt oxide particles must be reduced to metallic cobalt, which is done in the first part of the experiment using hydrogen atmosphere via the route: ${ }^{3,47} \mathrm{Co}_{3} \mathrm{O}_{4} \rightarrow \mathrm{CoO} \rightarrow \mathrm{Co}$, cf. Figure 3. This is clearly observed in the WAXS data (Figure 2), where the $\mathrm{Co}_{3} \mathrm{O}_{4}$ peaks disappear, $\mathrm{CoO}$ peaks [e.g., $\mathrm{CoO}$ (200) at $Q=2.95 \AA^{-1}$ ] appear and then decrease with temperature, and Co metal peaks appear. The Q-range of our WAXS measurements covers only a few Co metal diffraction peaks, making it difficult to distinguish hcp (hexagonal closepacked) or fcc (face-centered cubic) structures, since the measured peaks overlap either with each other or with a $\mathrm{CoO}$ peak. Some variations in $\alpha$, and thus $\Delta \alpha$, were observed during 

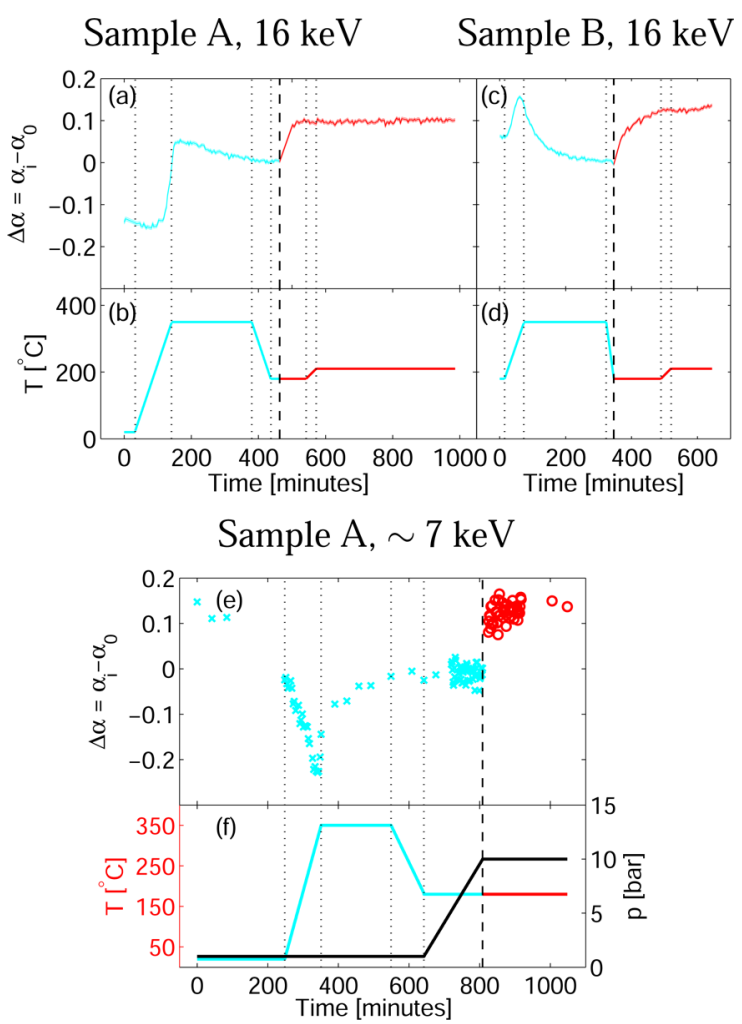

Figure 5. The results for $\Delta \alpha=\alpha_{i}-\alpha_{0}$ for the SAXS patterns measured under different conditions. In the $E=16 \mathrm{keV}$ experiment, atmospheric pressure was applied, while for the ASAXS experiment $(E \sim 7 \mathrm{keV})$, the pressure was increased to $10 \mathrm{bar}$ before the synthesis gas was let into the system. Top panels (a, c, e): The difference $\Delta \alpha$ as function of time. Bottom panels (b, d, f): The experimental conditions. Cyan shows reduction, using $\mathrm{H}_{2}$ as feed gas, and red shows reaction, using synthesis gas $\left(\mathrm{H}_{2}\right.$ and $\left.\mathrm{CO}\right)$ as feed gas. Notice the same sudden increase of $\alpha$ in all experiments when the synthesis gas is introduced; this behavior is thus independent of pressure.

the reduction, as seen in Figure 5, which is not surprising because of the restructuring from oxide to metal.

Most interestingly, we observe a significant increase in $\Delta \alpha$ as the synthesis gas $\left(\mathrm{H}_{2}\right.$ and $\left.\mathrm{CO}\right)$ is introduced: In the $16 \mathrm{keV}$ experiment, for both samples $\mathrm{A}$ and $\mathrm{B}, \alpha$ increases by $\sim 0.1$ (Figure 5a,c). The parameter values for $\alpha_{0}$ were found to be 4.2 and 4.1 for samples A and B, respectively. In the $7 \mathrm{keV}$ ASAXS experiment, only sample $\mathrm{A}$ was measured, and also here $\Delta \alpha$ increases to $\sim 0.1$ (Figure 5e), and $\alpha_{0}$ was found to be 4.0. The Porod slope $\alpha_{0}$ is thus $\approx 4$ in all the experiments for the reduced sample, and $\alpha>4$ after the gas change.

The support material, $\gamma$-alumina, was chosen because it is known to be structurally stable at least up to $750{ }^{\circ} \mathrm{C} .{ }^{48}$ The support had been calcined prior to these studies, and we do not expect it to change during the experiments reported here. While the alumina support may influence the absolute value of $\alpha$, we thus assume that changes in $\alpha$ are due to changes in the cobalt particles, which is also substantiated by the dominance of cobalt at these scattering angles.

An estimate of the width, $w$, of the transition between particle phase and the surrounding material can be made by plotting eq 3, varying the standard deviation of the Gaussian distribution $\sigma_{\text {surf }}$, and fitting the high $Q$ enveloping slope to the measured power-law slope. The width of the real-space Gaussian here used is $w=4 \sigma_{\text {surf }}$, and after convolution with the density function $\rho(r)$, this becomes the width of the transition between particle phase and the surrounding material. The change in $w$ before and after introducing synthesis gas was here estimated to be $\Delta w=6 \pm 3 \AA$.

The invariants $G^{*}$, cf. Equation 5, obtained from the $16 \mathrm{keV}$ experiment are plotted in Figure 6, where we observe a

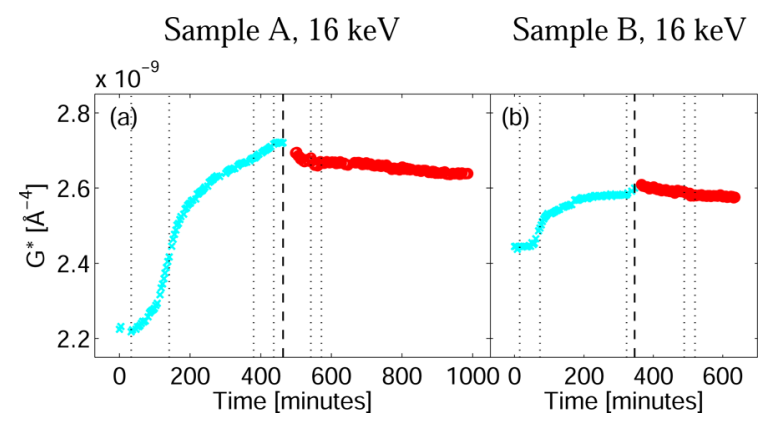

Figure 6. The scattering invariant estimated for (a) sample A and (b) sample B, calculated for all the measurements in the $16 \mathrm{keV}$ experiment. Cyan shows reduction, using $\mathrm{H}_{2}$ as feed gas, red shows reaction, using synthesis gas $\left(\mathrm{H}_{2}\right.$ and $\left.\mathrm{CO}\right)$ as feed gas. Sample B was already partially reduced at the start of the measurement series. The vertical dotted lines indicate changes in temperature settings, and the dashed vertical line indicate change of gas (see Figure $5 b, d$ for further details).

$21 \pm 2 \%$ increase of the invariant during the reduction for sample A. If calculating the invariant from a three-phase sample, first for the case where all the cobalt is in the $\mathrm{Co}_{3} \mathrm{O}_{4}$ state (cf. Figure 3) and then for it all being in the metallic phase $\left(\mathrm{Co}^{0}\right)$ and taking into account the relative volume changes, we estimate a $15-20 \%$ increase of $G$, which is mainly due to the significantly higher scattering length density of $\mathrm{Co}^{0}$ compared to $\mathrm{Co}_{3} \mathrm{O}_{4}$. The strong increase of the invariant observed during reduction is thus fully consistent with theory. During the reaction stage, the invariant is observed to decrease slightly, which will be discussed below.

\section{DISCUSSION}

As mentioned, the power-law exponent $\alpha$ is related to the surface structure and shape of the particles. Consequently, the sudden increase of $\alpha$ when the synthesis gas is introduced (Figure 5) suggests that $\mathrm{CO}$ affects the morphology of the cobalt particles. This is an interesting and potentially important observation, and possible underlying mechanisms, as schematically illustrated in Figure 7, will now be discussed systematically.

(i). Shape Change/Faceting. A hypothesis for the increase in $\alpha$ is that the particles change their shape to become, for example, more elliptical or faceted. However, as long as the ellipsoid remains a sharply defined 3D object, $\alpha$ should remain $\leq 4{ }^{36}$ Randomly oriented faceted particles would yield $\alpha<4$ due to the rods of scattering parallel to the facet normals, ${ }^{49}$ thus causing $\alpha$ to decrease, rather than the observed increase. This model therefore cannot account for our observations of $\alpha$ beyond 4 . The SAXS invariant should remain constant for this scenario, whereas what we measure is apparently a slight decrease with time during the reaction stage. From the SAXS analysis, we therefore find this hypothesis of shape change or faceting of the cobalt particles to be unlikely.

(ii). Surface Reconstruction and Relaxation. Surface reconstruction could be a realistic process explaining the sudden change in $\alpha$; as $\alpha$ increases beyond 4, this suggests that 
(i)

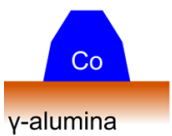

(iv)

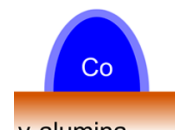

y-alumina (ii)

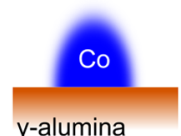

(v)

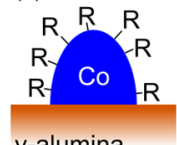

y-alumina (iii)

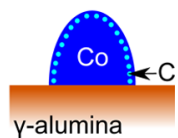

(vi)

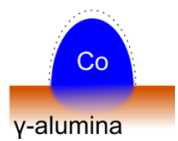

Figure 7. Schematic presentation of possible changes to the cobalt particles as the synthesis gas is introduced: (i) faceting, (ii) surface reconstruction and relaxation, (iii) formation of a subsurface carbon layer, (iv) surface reoxidation, (v) adsorbed monolayer of $\mathrm{CO}$ or a layer of hydrocarbons, and (vi) diffusion/interaction with the alumina support.

the Co nanoparticles, which are known from TEM measurements to be approximately spherical, ${ }^{15}$ assume a more graded, i.e., reduced density transition region from the core to the surrounding atmosphere. The length-scale of $6 \AA$ typically corresponds to two or three atomic layers, which is in agreement with $\mathrm{CO}$ adsorption and subsequent surface reconstruction. Also for this scenario the invariant should remain constant, and the discussion of the invariant analysis in the previous point (i) is also valid here.

(iii). A Subsurface Carbon Layer. This mechanism has been suggested by density-functional-theory (DFT) calculations ${ }^{50}$ and is also a possible explanation for increased $\alpha$, for the same reasons as the previous point (ii). This hypothesis is difficult to confirm or reject due to the low atomic mass of carbon compared to the other constituents of the sample. By estimating the invariant $G$ from eq 6 using four phases, Co + $\mathrm{Al}_{2} \mathrm{O}_{3}+$ pores $+\mathrm{C}$ (see Figure 3, where in this case $\mathrm{C}$ is the "other" phase), we find that an unrealistically large amount of $\mathrm{C}$ would have to be added in order to comprise the difference measured at the beginning and at the end of the reaction, cf. Figure 6. From the invariant analysis alone we cannot confirm or reject this hypothesis, and it is plausible that carbon intercalates into the Co lattice.

(iv). Surface Reoxidation. A frequently debated topic in cobalt-based FTS catalyst deactivation is the possible reoxidation of active cobalt sites during synthesis, due to the fact that water, the most abundant byproduct of FTS, is an oxidizing agent and thus may cause surface oxidation of the cobalt nanoparticles. ${ }^{43}$ However, it is possible to determine whether the conditions in the FTS reactor are predominantly reducing or oxidizing by mounting a partially reduced catalyst, where the $\mathrm{CoO}$ diffraction peaks were clearly visible, and exposing it to the FTS conditions. We have previously done this test ${ }^{13}$ and observed a slight decrease in the CoO peak intensities, indicating further reduction of the cobalt catalysts. We have also monitored the cobalt catalysts using XANES (Xray absorption near edge structure), which is sensitive to oxidation state, during the reaction for $6 \mathrm{~h}$ at $210^{\circ} \mathrm{C}$ and 18 bar ${ }^{13}$ and for $32 \mathrm{~h}$ at $220^{\circ} \mathrm{C}$ and $18 \mathrm{bar}^{3}$. No reoxidation could be detected in these XANES experiments, and it is unlikely that it would happen in the present study performed under similar experimental conditions. In the surface reoxidation scenario the invariant would be expected to decrease, and from the invariant analysis alone this hypothesis is thus considered possible, but, as discussed, the surface reoxidation is anyway unlikely due to the reducing sample environment.

(v). An Adsorbed Monolayer of CO or a Layer of Hydrocarbons. This is also unlikely as we have previously measured the catalytic activity at $180{ }^{\circ} \mathrm{C}$ and it has been found to be low. ${ }^{3}$ Notice that the sudden increase in $\alpha$ was observed for the catalyst samples at a temperature of $180{ }^{\circ} \mathrm{C}$, which is below the formation temperature of long hydrocarbons. Furthermore, we would not be able to detect a thin layer of adsorbed molecules using SAXS because of low contrast (cf. $\Delta \rho$ in eq 1) between the adsorbates and the surrounding medium. The difference in the invariant would in this case be too small to observe.

(vi). Diffusion or Interactions with the Alumina Support. We have previously measured diffusion of Co into the alumina support using XAS, finding that a minor fraction of cobalt atoms randomly occupy the tetrahedral vacancies of the alumina support. ${ }^{51}$ This diffusion would also cause a graded interface, now between the cobalt particles and the alumina support. The diffusion was observed before and after reduction by hydrogen at $450{ }^{\circ} \mathrm{C}$, meaning that the diffusion already takes place before the feed of synthesis gas starts. We have previously measured XAS from a rhenium-promoted $\mathrm{Co} / \mathrm{Al}_{2} \mathrm{O}_{3}$ sample during an in situ experiment, where we found that the Coaluminate formation increases gradually after the synthesis gas was introduced (the starting point of the FTS experiment) and during the induction period, i.e., until the FTS conversion rate had stabilized. However, when comparing this result to our SAXS results, we do not see further evolution of $\alpha$ after the changes related to introducing the synthesis gas, cf. Figure 5a, and consequently, the aluminate formation apparently does not give a significant contribution to the SAXS signal. The invariant has been estimated to decrease as Co diffuses into $\mathrm{Al}_{2} \mathrm{O}_{3}$ and forms $\mathrm{CoAl}_{2} \mathrm{O}_{4}$. Even if the mechanism of diffusion into the alumina is probably not the cause of the sudden increase of $\alpha$ after gas-change, diffusion may still be happening on a longer time scale, and the present work does not contradict ref 51 .

Although scanning tunneling microscopy cannot be employed under realistic conditions for FTS due to vacuum requirements, it can still be used for studying the effect of adsorbing molecules onto surfaces. Venvik et al. ${ }^{18,19}$ studied the effect of exposing specific common Co surfaces, $\mathrm{Co}(11 \overline{2} 0)$ and $\mathrm{Co}(10 \overline{1} 2)$, to $\mathrm{CO}$ and showed that $\mathrm{CO}$ caused the Co atoms to migrate and the surface to undergo reconstruction. CO-induced surface reconstruction of cobalt is also in agreement with the hypothesis made by Schulz et $\mathrm{al}^{4}$ that the reaction of $\mathrm{CO}$ with the Co surface can be assumed to induce surface restructuring. Since the hypotheses of aluminate formation, shape change, surface oxidation, and monolayer of $\mathrm{CO}$ or hydrocarbons all seem unlikely, it appears that the most likely reason for the change in $\alpha$ is that the presence of $\mathrm{CO}$ gas induces Co atoms to migrate and the surface to rearrange, possibly with a subsurface layer of carbon, effectively forming a lower density particle exterior.

\section{CONCLUSION}

Using SAXS, we have measured Co nanoparticles for FischerTropsch synthesis in situ under realistic catalysis conditions (feed of $\mathrm{H}_{2}$ and $\mathrm{CO}$ gases, heating to $210{ }^{\circ} \mathrm{C}$, pressure up to 10 bar, and $\gamma$-alumina support). The Porod exponent of the scattering patterns was found to change markedly upon switching from reduction to synthesis gas, which is interpreted as a change of the surface of the cobalt catalyst particles. Several 
possible models for explaining the observed data have been discussed, and we argue that a likely reason for the observations is that adsorbed $\mathrm{CO}$ molecules cause the Co surface atoms to migrate, thus causing the transition-width between the Co particle bulk phase and the surrounding medium to increase, effectively making the particles less well-defined. Knowledge about the particle surfaces during catalytic reactions is important because it is where catalysis takes place. We have shown that it is possible to measure surface-related changes in industrially relevant nanoparticles on a porous support, under experimentally challenging in situ conditions. To obtain a better understanding of catalysis, being able to retrieve structural information well beyond simplified model systems will become increasingly important.

\section{AUTHOR INFORMATION}

\section{Corresponding Author}

*E-mail: dag.breiby@ntnu.no. Phone: +47 73593594.

\section{Notes}

The authors declare no competing financial interest.

\section{ACKNOWLEDGMENTS}

Parts of this research were carried out at the light source DORIS III at DESY, a member of the Helmholtz Association (HGF). We would like to thank Stephan Botta and Jan Rubeck for assistance at beamline B1. The Research Council of Norway (SYNKROTRON programme), The Danish Council for Independent Research | Natural Sciences, DANSCATT (Danish Centre for the use of Synchrotron X-ray and Neutron facilities), and ELISA (EU support of access to synchrotrons/ FELs in Europe), are gratefully acknowledged for financial support.

\section{REFERENCES}

(1) Chorkendorff, I.; Niemantsverdriet, J. Concepts of Modern Catalysis and Kinetics, 2nd ed.; Wiley-VCH: Weinheim, 2007; Section 8.3.2

(2) Oukaci, R.; Singleton, A. H.; Goodwin, J. G. Comparison of Patented Co F-T Catalysts using Fixed-Bed and Slurry Bubble Column Reactors. Appl. Catal., A 1999, 186, 129-144.

(3) Tsakoumis, N. E.; Voronov, A.; Rønning, M.; van Beek, W.; Borg, Ø.; Rytter, E.; Holmen, A. Fischer-Tropsch Synthesis: An XAS/ XRPD Combined In Situ Study from Catalyst Activation to Deactivation. J. Catal. 2012, 291, 138-148.

(4) Schulz, H.; Nie, Z.; Ousmanov, F. Construction of the FischerTropsch Regime with Cobalt Catalysts. Catal. Today 2002, 71, 351360.

(5) Wilson, J.; de Groot, C. Atomic-Scale Restructuring in HighPressure Catalysis. J. Phys. Chem. 1995, 99, 7860-7866.

(6) Beitel, G. A.; Laskov, A.; Oosterbeek, H.; Kuipers, E. W. Polarization Modulation Infrared Reflection Absorption Spectroscopy of $\mathrm{CO}$ Adsorption on $\mathrm{Co}(0001)$ under a High-Pressure Regime. J. Phys. Chem. 1996, 100, 12494-12502.

(7) Geerlings, J.; Zonnevylle, M.; de Groot, C. Studies of the Fischer-Tropsch Reaction on Co(0001). Surf. Sci. 1991, 241, 302314.

(8) Geerlings, J.; Zonnevylle, M.; de Groot, C. Structure Sensitivity of the Fischer-Tropsch Reaction on Cobalt Single Crystals. Surf. Sci. 1991, 241, 315-324.

(9) Hilmen, A. M.; Schanke, D.; Holmen, A. Reoxidation of Supported Cobalt Fischer-Tropsch Catalysts. In Natural Gas Conversion IV; de Pontes, M., Espinoza, R. L., Nicolaides, C. P., Scholtz, J. H., Scurrell, M. S., Eds.; Elsevier: Amsterdam, 1997; Vol. 107, pp 237-242.
(10) Hilmen, A.; Schanke, D.; Hanssen, K.; Holmen, A. Study of the Effect of Water on Alumina Supported Cobalt Fischer-Tropsch Catalysts. Appl. Catal., A 1999, 186, 169-188.

(11) Curtis, V.; Nicolaides, C.; Coville, N.; Hildebrandt, D.; Glasser, D. The Effect of Sulfur on Supported Cobalt Fischer-Tropsch Catalysts. Catal. Today 1999, 49, 33-40.

(12) Niemelä, M. K.; Krause, A. O. I. The Long-Term Performance of $\mathrm{Co} / \mathrm{SiO}_{2}$ Catalysts in $\mathrm{CO}$ Hydrogenation. Catal. Lett. 1996, 42, 161-166.

(13) Rønning, M.; Tsakoumis, N. E.; Voronov, A.; Johnsen, R. E.; Norby, P.; van Beek, W.; Borg, Ø.; Rytter, E.; Holmen, A. Combined $\mathrm{XRD}$ and XANES Studies of a Re-Promoted $\mathrm{Co} / \gamma-\mathrm{Al}_{2} \mathrm{O}_{3}$ Catalyst at Fischer-Tropsch Synthesis Conditions. Catal. Today 2010, 155, 289295.

(14) Borg, Ø.; Dietzel, P.; Spjelkavik, A.; Tveten, E.; Walmsley, J.; Diplas, S.; Eri, S.; Holmen, A.; Rytter, E. Fischer-Tropsch Synthesis: Cobalt Particle Size and Support Effects on Intrinsic Activity and Product Distribution. J. Catal. 2008, 259, 161-164.

(15) Dehghan, R.; Hansen, T.; Wagner, J.; Holmen, A.; Rytter, E.; Borg, Ø.; Walmsley, J. In-situ Reduction of Promoted Cobalt Oxide Supported on Alumina by Environmental Transmission Electron Microscopy. Catal. Lett. 2011, 141, 754-761.

(16) Jacobs, G.; Das, T. K.; Patterson, P. M.; Li, J.; Sanchez, L.; Davis, B. H. Fischer-Tropsch Synthesis XAFS: XAFS Studies of the Effect of Water on a Pt-Promoted $\mathrm{Co} / \mathrm{Al}_{2} \mathrm{O}_{3}$ Catalyst. Appl. Catal., A 2003, 247, 335-343.

(17) van Berge, P.; van de Loosdrecht, J.; Barradas, S.; van der Kraan, A. Oxidation of Cobalt Based Fischer-Tropsch Catalysts as a Deactivation Mechanism. Catal. Today 2000, 58, 321-334.

(18) Venvik, H. J.; Borg, A.; Berg, C. Formation of the CO-induced $(3 \times 1)$ Surface Structure on $\mathrm{Co}(11 \overline{2} 0)$ Studied by STM. Surf. Sci. 1998, 397, 322-332.

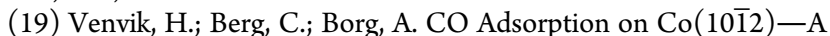
STM Study. Surf. Sci. 1998, 402-404, 57-61.

(20) Feigin, L.; Taylor, G. W.; Svergun, D. Structure Analysis by Small-Angle X-ray and Neutron Scattering; Plenum Press: New York, 1987.

(21) Wang, W.; Zhang, K.; Cai, Q.; Mo, G.; Xing, X. Q.; Cheng, W. D.; Chen, Z. J.; Wu, Z. H. Real-Time SAXS and Ultraviolet-Visible Spectral Studies on Size and Shape Evolution of Gold Nanoparticles in Aqueous Solution. Eur. Phys. J. B 2010, 76, 301-307.

(22) Giovanetti, L. J.; Ramallo-López, J. M.; Foxe, M.; Jones, L. C.; Koebel, M. M.; Somorjai, G. A.; Craievich, A. F.; Salmeron, M. B.; Requejo, F. G. Shape Changes of Pt Nanoparticles Induced by Deposition on Mesoporous Silica. Small 2012, 8, 468-473.

(23) Kehres, J.; Andreasen, J. W.; Krebs, F. C.; Molenbroek, A. M.; Chorkendorff, I.; Vegge, T. Combined in Situ Small- and Wide-Angle X-ray Scattering Studies of $\mathrm{TiO}_{2}$ Nanoparticle Annealing to 1023K. J. Appl. Crystallogr. 2010, 43, 1400-1408.

(24) Preux, O.; Durand, D.; Huet, A.; Conway, J. A Two-State Cooperative Expansion Converts the Procapsid Shell of Bacteriophage T5 into a Highly Stable Capsid Isomorphous to the Final Virion Head. J. Mol. Biol. 2013, 425, 1999-2014.

(25) Braun, A.; Ilavsky, J.; Dunn, B. C.; Frank, E.; Huffman, G. P. Ostwald Ripening of Cobalt Precipitates in Silica Aerogels? An UltraSmall-Angle X-ray Scattering Study. J. Appl. Crystallogr. 2003, 38, 132-138.

(26) Renaud, G.; Lazzari, R.; Revenant, C.; Barbier, A.; Noblet, M.; Ulrich, O.; Leroy, F.; Jupille, J.; Borensztein, Y.; Henry, C.; et al. RealTime Monitoring of Growing Nanoparticles. Science 2003, 300, 14161419.

(27) Hoydalsvik, K.; Barnardo, T.; Winter, R.; Haas, S.; Tatchev, D.; Hoell, A. Yttria-Zirconia Coatings Studied by Grazing-Incidence Small-Angle X-ray Scattering During in Situ Heating. Phys. Chem. Chem. Phys. 2010, 12, 14492-14500.

(28) Tyo, E. C.; Yin, C.; Di Vece, M.; Qian, Q.; Kwon, G.; Lee, S.; Lee, B.; DeBartolo, J. E.; Seifert, S.; Winans, R. E.; et al. Oxidative Dehydrogenation of Cyclohexane on Cobalt Oxide $\left(\mathrm{Co}_{3} \mathrm{O}_{4}\right)$ Nano- 
particles: The Effect of Particle Size on Activity and Selectivity. ACS Catal. 2012, 2, 2409-2423.

(29) Ferguson, G. A.; Yin, C.; Kwon, G.; Tyo, E. C.; Lee, S.; Greeley, J. P.; Zapol, P.; Lee, B.; Seifert, S.; Winans, R. E.; et al. Stable Subnanometer Cobalt Oxide Clusters on Ultrananocrystalline Diamond and Alumina Supports: Oxidation State and the Origin of Sintering Resistance. J. Phys. Chem. C 2012, 116, 24027-24034.

(30) Revenant, C.; Leroy, F.; Lazzari, R.; Renaud, G.; Henry, C. Quantitative Analysis of Grazing Incidence Small-Angle X-ray Scattering: Pd/MgO(001) Growth. Phys. Rev. B 2004, 69, 035411.

(31) Pedersen, J. S. Analysis of Small-angle Scattering Data from Colloids and Polymer Solutions: Modeling and Least-Squares Fitting. Adv. Colloid Interface Sci. 1997, 70, 171-210.

(32) Haug, J.; Kruth, H.; Dubiel, M.; Hofmeister, H.; Haas, S.; Tatchev, D.; Hoell, A. ASAXS Study on the Formation of Core-Shell $\mathrm{Ag} / \mathrm{Au}$ Nanoparticles in Glass. Nanotechnology 2009, 20, 505705.

(33) Als-Nielsen, J.; McMorrow, D. Elements of Modern X-ray Physics, 2nd ed.; Wiley: Chichester, UK, 2011.

(34) Guinier, A.; Fournet, G. Small-Angle Scattering of X-rays; Wiley: New York, 1955.

(35) Vainio, U. Small-Angle X-ray Scattering. In X-ray Diffraction: Modern Experimental Techniques; Seech, O. H., Murphy, B. M., Eds.; Pan Stanford Publishing Pte Ltd: Singapore, 2014; Chapter 4.

(36) Schmidt, P. W. Small-Angle Scattering Studies of Disordered, Porous and Fractal Systems. J. Appl. Crystallogr. 1991, 24, 414-435.

(37) Schmidt, P. W.; Avnir, D.; Levy, D.; Hohr, A.; Steiner, M.; Roll, A. Small-Angle X-ray Scattering from the Surfaces of Reversed-Phase Silicas: Power-Law Scattering Exponents of Magnitudes Greater than Four. J. Chem. Phys. 1991, 94, 1474-1479.

(38) Stieger, M.; Pedersen, J. S.; Lindner, P.; Richtering, W. Are Thermoresponsive Microgels Model Systems for Concentrated Colloidal Suspensions? A Rheology and Small-Angle Neutron Scattering Study. Langmuir 2004, 20, 7283-7292.

(39) Cheung, Y. W.; Stein, R. S.; Lin, J. S.; Wignall, G. D. SmallAngle Scattering Investigations of Poly $(\varepsilon$-caprolactone)/Polycarbonate Blends. 2. Small-Angle X-ray and Light Scattering Study of Semicrystalline/Semicrystalline and Semicrystalline/Amorphous Blend Morphologies. Macromolecules 1994, 27, 2520-2528.

(40) Tatchev, D. Structure Analysis of Multiphase Systems by Anomalous Small-Angle X-ray Scattering. Philos. Mag. 2008, 88, $1751-1772$.

(41) Vainio, U.; Lauten, R. A.; Haas, S.; Svedström, K.; Veiga, L. S. I.; Hoell, A.; Serimaa, R. Distribution of Counterions around Lignosulfonate Macromolecules in Different Polar Solvent Mixtures. Langmuir 2012, 28, 2465-2475.

(42) Andreasen, J. W.; Rasmussen, O.; Feidenhans'l, R.; Berg Rasmussen, F.; Christensen, R.; Molenbroek, A. M.; Goerigk, G. An In Situ Cell for Small-Angle Scattering Experiments on Nano-Structured Catalysts. J. Appl. Crystallogr. 2003, 36, 812-813.

(43) Tsakoumis, N. E.; Rønning, M.; Borg, Ø.; Rytter, E.; Holmen, A. Deactivation of Cobalt Based Fischer-Tropsch Catalysts: A Review. Catal. Today 2010, 154, 162-182.

(44) Kraft, P.; Bergamaschi, A.; Bronnimann, C.; Dinapoli, R.; Eikenberry, E. F.; Graafsma, H.; Henrich, B.; Johnson, I.; Kobas, M.; Mozzanica, A.; et al. Characterization and Calibration of PILATUS Detectors. IEEE Trans. Nucl. Sci. 2009, 56, 758-764.

(45) Zhang, F.; Ilavsky, J.; Long, G. G.; Quintana, J. P. G.; Allen, A. J.; Jemian, P. R. Glassy Carbon as an Absolute Intensity Calibration Standard for Small-Angle Scattering. Metall. Mater. Trans. A 2010, 41A, 1151-1158.

(46) Simon, J. P.; Lyon, O. Anomalous Small Angle X-ray Scattering in Materials Science. In Resonant Anomalous X-ray Scattering: Theory and Applications; Materlik, C., Sparks, C. J., Fischer, K., Eds.; Elsevier Science V.B.: North-Holland, 1994; pp 305-322.

(47) Bulavchenko, O.; Cherepanova, S.; Malakhov, V.; Dovlitova, L.; Ishchenko, A.; Tsybulya, S. In Situ XRD Study of Nanocrystalline Cobalt Oxide Reduction. Kinet. Catal. 2009, 50, 192-198.

(48) Paglia, G.; Buckley, C. E.; Rohl, A. L.; Hart, R. D.; Winter, K.; Studer, A. J.; Hunter, B. A.; Hanna, J. V. Boehmite Derived $\gamma$-Alumina
System. 1. Structural Evolution with Temperature, with the Identification and Structural Determination of a New Transition Phase, $\gamma^{\prime}$-Alumina. Chem. Mater. 2004, 16, 220-236.

(49) Lazzari, R. Grazing Incidence Small-Angle X-ray Scattering from Nanostructures. In X-ray and Neutron Reflectivity: Principles and Applications; Daillant, J., Gibaud, A., Eds.; Springer: Berlin, 2009; Chapter 7.

(50) Zonnevylle, M.; Geerlings, J.; van Santen, R. Conversion of Surface Carbidic to Subsurface Carbon on Cobalt (0001): A Theoretical Study. Surf. Sci. 1990, 240, 253-262.

(51) Moen, A.; Nicholson, D. G.; Ronning, M.; Emerich, H. In Situ $\mathrm{X}$-ray Absorption Spectroscopic Studies at the Cobalt K-Edge on an $\mathrm{Al}_{2} \mathrm{O}_{3}$-Supported Rhenium-Promoted Cobalt Fischer-Tropsch Catalyst. Comparing Reductions in High and Low Concentration Hydrogen. J. Mater. Chem. 1998, 8, 2533-2539. 